\title{
Generic Reliability Evaluation Method for Industrial Grids with Variable Frequency Drives
}

\author{
Rongrong Yü, Yao Chen ${ }^{1}$, Jiuping Pan ${ }^{2}$, Richard W Vesel ${ }^{3}$ \\ ${ }^{1}$ Power Systems, ABB Corporate Research Beijing, China \\ ${ }^{2}$ Power Technologies, ABB Corporate Research, Raleigh, USA \\ ${ }^{3}$ EBoP Energy Efficiency, ABB Power Generation, Wickliffe, USA \\ Email: rongrong.yu@cn.abb.com, cathy-yao.chen@cn.abb.com, jiuping.pan@us.abb.com, richard.w.vesel@us.abb.com
}

Received January, 2013

\begin{abstract}
The paper presents a reliability evaluation method based on fault tree analysis with set theory and minimal cut set as core algorithm, which can be used to evaluate the reliability for industrial grids with wide application of variable frequency drives. The working principle is introduced firstly, based on which the method development considering different system topology designs, backup solutions and redundancy mechanisms are analyzed in details. In the end the proposed method is applied to two cases to show the reliability performance of system with variable frequency drives. The proposed method is also suitable for analyzing the reliability performance of industrial grids with other types of power electronic converter technology.
\end{abstract}

Keywords: Fault Tree Analysis; Minimal Cut Set; Reliability; Industrial Grids; Variable Frequency Drives

\section{Introduction}

One of the most essential elements contributing to quality of industrial grids is design of its electrification scheme. Nowadays in order to increase system efficiency and reduce energy consumption losses, power electronic devices like Variable Frequency Drives (VFD) are widely applied in both power generation and distribution grids. With introduction of power electronic devices, reliability of the original system topology may be influenced. However, conventional reliability evaluation method as in [1] is designed without considering VFD. As a result, it's worthwhile finding a generic approach to evaluate the reliability of industrial grids with VFDs or other types of power electronic converter technology to appraise VFD impact and propose improvement solution.

Most commonly used reliability study methods for power system include Reliability Block Diagram (RBD) [2], Markov chain [3], Monte Carlo simulation [4] and Fault Tree Analysis (FTA) [5]. Considering the feature of industrial grids, for example, multi-input and multi-output, large quantity of components, known electrification scheme, desiring to identify key component(s) with biggest impact on the entire system reliability, as well as for the purpose of reflecting reliability trend after introducing VFDs, FTA is chosen in this paper to develop a generic evaluation algorithm. Minimal cut set is embedded as basic failure event identification way in FTA. In the following parts of the paper, firstly, generic working principle of using FTA to evaluate reliability for industrial plants is introduced; Secondly, detailed calculation procedures with minimum cut set and set theory considering different system topology designs, backup solutions and redundancy mechanisms are analyzed in details; thirdly, the proposed method is demonstrated respectively with two case studies.

\section{Principle Analysis of FTA}

\subsection{Working Principle}

The main steps of FTA are described in Figure 1, starting from network topology definition, e.g. single-bus or dual- bus power circuit topology; to top event identification, which usually refers to system power supply interruption; to minimum cut set categorization, to come out with groups of basic failure events that will lead to the top event; and finally to reliability indices calculation. The final indicator will be Expected Energy Not Supplied (EENS), i.e. the undesired energy losses due to reliability issues.

\subsection{Input and Output Indices}

According to the basic theory of FTA, following component reliability data is required as input information to quantify the reliability indices for each failure event: total failure rate, active failure rate, repair time, switching time, stuck probability, maintenance rate and main- 
tenance time [1].

Output indices to measure the reliability performance for a given system include: failure rate, outage duration, average repair time and EENS.

\section{Reliability Evaluation for Single-bus Bar System}

A typical single-bus bar system can be separated into two parts for reliability analysis: one is power supply part as in Figure 2(a), including a main path consisting of the main transformer in series with a normally close breaker, and a spare path consisting of the backup transformer in series with a normally open breaker; the other is power consumption part as in Figure 2(b), including a number of branches, each consisting of motor, individual VFD, line-side transformer and normally close breaker. The reason for this separation is that the consequences of failure occurrence in these two parts reflecting by EENS are different from the whole system point of view.

\subsection{Reliability Evaluation for Power Supply Part}

In this section, reliability evaluation method for power supply part is firstly presented. By defining the following two events, A \& B:

Event A - failure event that can be eliminated by closing the spare path;

Event B - event that the spare path is available before having the components of failure event a restored back to service.

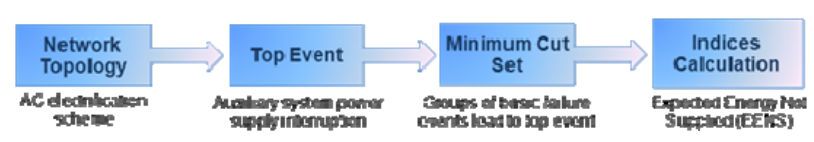

Figure 1. Main steps of FTA.

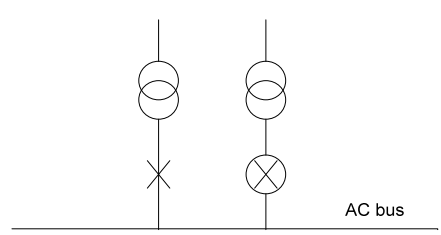

(a) Power supply part

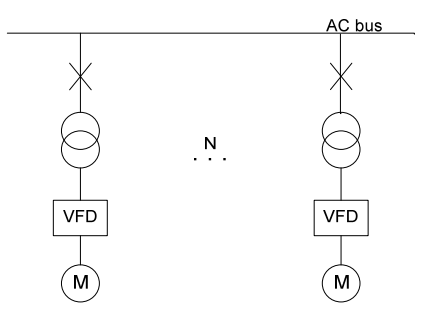

(b) Power consumption part

Figure 2. Reliability evaluation for single bus bar topology.
According to total probability formula, outage duration of event A can be calculated by:

$$
\begin{aligned}
u_{A}= & u(A \mid B)\left[1-\left(u_{e} / 8760+P_{s}\right)\right] \\
& +u(A \mid \bar{B})\left(u_{e} / 8760+P_{s}\right)
\end{aligned}
$$

where $u(A \mid B)$ is annual outage duration caused by event $A \mid B$, indicating that the spare path is always available when needed; $u(A \mid \bar{B})$ is annual outage duration of event $A \| \bar{B}$ indicating the spare path is unavailable to restore service; $u_{\varepsilon}$ is the equivalent outage duration of the spare path; $P_{s}$ is the stuck probability of breaker; 8760 indicates the hours per year. It should be noticed that besides the failure probability, the stuck breaker might also lead to the undesired outage.

Two steps are needed to utilize (1) to obtain reliability indices of power supply part as in Figure 2(a), which is exactly the two items of (1). The unavailability of the spare path comprises two cases: forced outage and maintenance, which both make it unable to replace the failed main path to continue the power supply. Integrating all of them together, we totally consider three possible cases, where overlapped maintenance for both main path and spare path is not considered here.

- Main path failure plus spare path failure;

- Main path failure plus spare path maintenance;

- Main path maintenance plus spare path failure.

Based on the analysis above, we can obtain the outage duration $u_{G 1}$ caused by the main transformer:

$$
\begin{aligned}
u_{A 1}= & \lambda r_{s}\left[1-\left(u_{e} / 8760+u_{e}^{\prime \prime} / 8760+P_{s}\right)\right] \\
& +\lambda^{\prime \prime} r^{\prime \prime}\left(u_{e} / 8760+P_{s}\right)
\end{aligned}
$$

where $\lambda$ and $\lambda^{\mathrm{n}}$ are the failure rate and maintenance rate of main transformer respectively; $r_{s}$ and $r^{n}$ are switching time and maintenance time of main transformer; $u_{e}$ is the equivalent outage duration of the spare path due to failure; $u_{e}^{n}$ is the equivalent outage duration of the spare path due to scheduled maintenance. By using the same method we can calculate the outage duration $u_{A 2}$ caused by the normally close circuit breaker, and then obtain the overall outage duration of power supply path $\tau_{A 1}+\tau_{A 2}$ with the unit of hours per year. Assuming the power output of such system is $P_{a}$, EENS can be calculated by $P_{o}\left(\tau_{A 1}+\tau_{A 2}\right)$ with unit of $\mathrm{MWh} / \mathrm{y}$. The higher the EENS is, the worse the reliability will be.

\subsection{Reliability Evaluation for Power Consumption Part}

In this section, reliability evaluation method suitable for the power consumption part will be given. Assuming there are $\mathrm{N}$ branches, and $\mathrm{N}-1$ redundancy mechanism is adopted, i.e. no output loss will be caused by removal of single motor branch, by iteratively using the total probability formula for N-1 times and integrating all the re- 
sults together, the final EENS caused by the power consumption part can be obtained (Detailed calculation procedures are ignored here).

$$
\text { EENS }=\sum_{i=1}^{N-1} P_{0} * \frac{1}{N-1} * u *(N-i) * p *(1-p)^{i-1}
$$

where $u$ and $p$ refer to the outage duration and the outage probability for each motor branch.

\section{Reliability Evaluation for Dual-bus Bar System}

A typical dual-bus bar system is shown in Figure 3, which can also be separated into two parts for reliability analysis: one is power supply part including two bus bars as in Figure 3(a), each supplied by a transformer in series with a normally close circuit breaker, and with a normally open circuit breaker in between; the other is power consumption part including a number of branches as in Figure 3(b), each consisting of motor, VFDs, lineside transformer and normally close (or normally open) circuit breaker.

\subsection{Reliability Evaluation for Power Supply Part}

In this section, reliability evaluation method for power supply part is firstly presented. Different with single bus bar topology, Set Theory should be introduced to evaluate the reliability of dual (or multiple buses) system. Assume event $\mathrm{A}$ is outage of bus bar 1 and event $\mathrm{B}$ is outage of the bus bar 2. As shown in Figure 4, the crossing section of set $A$ and set $B$ represents the occurrence of overlapping outage.

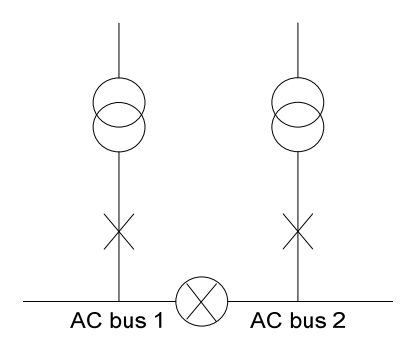

(a) Power supply part

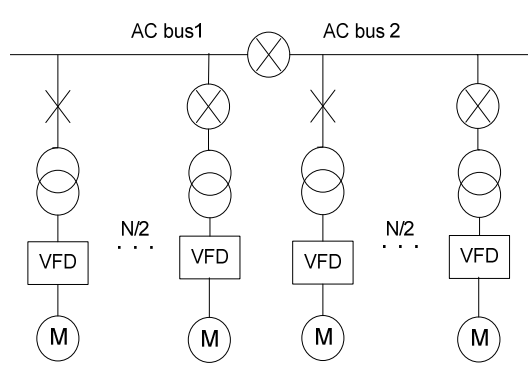

(b) Power consumption part

Figure 3. Reliability evaluation for dual bus bar topology.

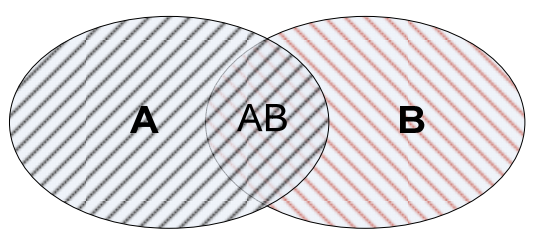

Figure 4. Set theory based busbar loss.

Based on the set definition and the total probability formula introduced above, outage duration of busbars can be obtained using equation below:

$$
\begin{aligned}
u_{\text {busbar }} & =u(A \bar{B})+u(B \bar{A})+u(A B) \\
& =u(A \mid \bar{B}) P(\bar{B})+u(B \mid \bar{A}) P(\bar{A})+u(A \mid B) P(B)
\end{aligned}
$$

where, $u(A \bar{B})$ is the duration of bus bar 1 outage while bus bar 2 in service; $u(B \bar{A})$ is the duration of bus bar 2 outage while bus bar 1 in service, $u(A \bar{B})=u(B \bar{A})$ if both bus bars are symmetrical; $u(A B)$ is the duration of bus bar 1 and 2 both fail. $P(\bar{A})$ and $P(\bar{B})$ are the normal operation probability of bus bar 1 and 2 ; $P(\bar{A})=P(\bar{B})$ if both bus bars are symmetrical.

The EENS of dual-bus system power supply part can be therefore calculated:

$$
\begin{aligned}
\text { EENS }_{\text {busbar }}= & P_{L}(A \bar{B}) u(A \bar{B})+P_{L}(B \bar{A}) u(B \bar{A}) \\
& +P_{L}(A B) u(A B)
\end{aligned}
$$

where, $P_{L}(A \bar{B})$ is the power loss in case of failure event $A \bar{B}$, namely one bus bar outage while the other bus bar in service, $P_{L}(A \bar{B})=P_{L}(B \bar{A})$ if both bus bars are symmetrical; $P_{L}(A B)$ is the system overall power loss.

\subsection{Reliability Evaluation for Power Consumption Part}

In this section, reliability evaluation method suitable for the power consumption part will be given. Assuming there are totally $\mathrm{N}$ motor branches connected to bus bar 1 and 2 ; and $\mathrm{N}+2$ redundancy mechanism is adopted, i.e. two standby branches (regardless of in the same or separate bus bars) can be switched in to maintain the output in case of the failure or maintenance event of any branch in operation, the outage duration caused by the power consumption part can be calculated as follows:

$$
\begin{aligned}
\text { umotor }_{=} & C_{N}^{1} P^{1}(1-P)^{N-1} u^{*} \\
& +\sum_{i=2}^{N} C_{N}^{i} P^{i}(1-P)^{N-i}\left[(i-2) u+2 u^{*}\right]
\end{aligned}
$$

where, $P$ is probability of each motor branch outage (considering both failure and maintenance); $u$ is outage duration of each motor branch without standby branch; $u^{*}$ is outage duration of each motor branch with standby branch. Assume $P_{m}$ is the rated power of the motor branch, the EENS of power consumption part can be calculated by $P_{m} * u_{\text {mator }}$. 


\section{Case Studies}

\subsection{Power Plant Auxiliary System}

Figure 5(a) shows a simplified Single Line Diagram (SLD) of a solar thermal power plant auxiliary system [6], with both main path and spare path for the power supply part, and totally 13 motor branches with VFDs for the power consumption part. The motor branches can be further categorized into three groups as indicated in the figure. The three groups of motors respectively take responsibility for driving pumps with three types of functionality:

- $6 \mathrm{x}$ heat transfer fluid pumps to pump the oil from the cold pipes through solar field to the hot pipes.

- $4 \mathrm{x}$ cold tank pumps to pump the cold molten salt from the cold tank through salt-HTF heat exchanger to the hot tank, to store extra solar energy during daytime.

- $3 \mathrm{x}$ hot tank pumps to pump the hot molten salt from the hot tank through salt-HTF heat exchanger to the cold tank, to discharge energy for power generation after sunset.

- For each group, N-1 redundancy mechanism is adopted.

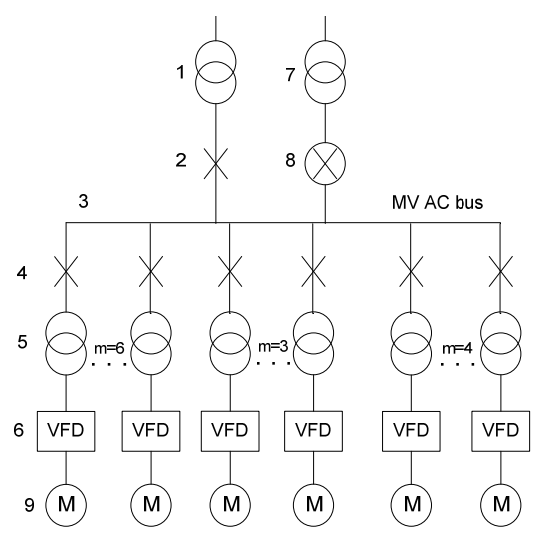

(a) Simplified SLD of power plant auxiliary system

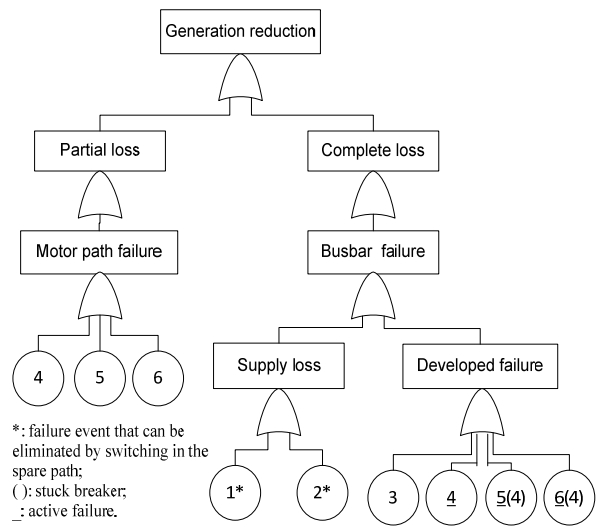

(b) Fault tree of power plant auxiliary system

Figure 5. Reliability case study for power plant auxiliary system..
By defining the generation reduction as the top failure event, the fault tree of the given system can be drawn as shown in Figure 5(b). The analysis is commenced by calculating reliability indices of each load point in the electrical auxiliary system, e.g. load point 3 (bus bar) that will lead to complete loss of generation, and load point 9 (auxiliary motor) that will lead to partial loss of generation.

Assuming the power generation capacity is $50 \mathrm{MW}$, the overall reliability indices for the single bus auxiliary system can be calculated as shown in Table 1. It can be observed that, the EENS with regard to the complete generation loss is mainly caused by power supply part, which is $33.67 \mathrm{MWh}$ per year; the EENS with regard to the partial generation loss caused by the motor branches is $4.14 \mathrm{MWh}$ per year. Totally, for the $50 \mathrm{MW}$ power plant, EENS caused by VFD based auxiliary system is $37.81 \mathrm{MWh} / \mathrm{y}$. It can also be seen from Table 1 that generation loss caused by VFD is about $5.33 \mathrm{~h} / \mathrm{y}$ (for each motor branch), corresponding to $3.15 \mathrm{WMh} / \mathrm{y}$ (13 motor branches), which accounts for over $8 \%$ of the overall EENS. One possible measure to further improve reliability is to utilize dual-bus topology which will be exemplified in the next section.

\subsection{Water Pumping Station}

Figure 6(a) shows a simplified SLD of a water pumping station, with two bus bars in the power supply part, and totally 8 motor branches with VFDs for the power consumption part. $\mathrm{N}+2$ redundancy mechanism is adopted, with two standby motor branches connected to the bus bars via normally open circuit breakers.

By defining the shortage of pumped water to be the top event, the fault tree of the given system can be drawn as shown in Figure 6(b), consisting of two groups of failure events: motor branch loss, and bus bar loss.

Table 1. Reliability indices for single bus power plant auxiliary system.

\begin{tabular}{|c|c|c|c|c|}
\hline \multirow{2}{*}{\multicolumn{2}{|c|}{ Failure event }} & \multicolumn{3}{|c|}{ Forced outages overlapping maintenance } \\
\hline & & $\lambda / \mathrm{y}$ & $\mathrm{u}(\mathrm{h} / \mathrm{y})$ & $\mathrm{r}(\mathrm{h})$ \\
\hline \multirow{6}{*}{ Complete loss } & $1 *$ & 0.52 & 0.43 & 0.83 \\
\hline & $2 *$ & 0.26 & 0.09 & 0.33 \\
\hline & 3 & 0.01 & 0.05 & 10.00 \\
\hline & $\underline{4}$ & 0.01 & 0.01 & 1.00 \\
\hline & $\underline{5}(4)$ & 0.00 & 0.00 & 1.00 \\
\hline & $\underline{6}(4)$ & 0.00 & 0.00 & 1.00 \\
\hline \multirow{2}{*}{\multicolumn{2}{|c|}{$\begin{array}{l}\text { Total (complete loss) } \\
\text { EENS }_{\text {complete }}(\mathrm{MWh} / \mathrm{y})\end{array}$}} & 0.89 & 0.67 & 0.76 \\
\hline & & \multicolumn{3}{|c|}{33.67} \\
\hline \multirow{3}{*}{ Partial loss } & 4 & 0.26 & 5.24 & 20.00 \\
\hline & 5 & 0.25 & 1.01 & 3.98 \\
\hline & 6 & 1.08 & 4.32 & 4.00 \\
\hline \multicolumn{2}{|c|}{$\mathrm{EENS}_{\text {partial }}(\mathrm{MWh} / \mathrm{y})$} & \multicolumn{3}{|c|}{4.14} \\
\hline \multicolumn{2}{|c|}{ EENS (MWh/y) } & \multicolumn{3}{|c|}{37.81} \\
\hline
\end{tabular}

Note: Bold data means it should be multiplied with 13 (number of motors) before summing. 
Using the Set Theory, the basic events leading to the bus bar failure can be specified. Assuming the power capacity of each motor is $4.095 \mathrm{MW}$, the overall reliability indices for the dual bus water pumping station can be calculated as shown in Table 2. The overall energy loss in this case is $9.97 \mathrm{MWh} / \mathrm{y}$, the majority of which comes from losses on bus bars. Losses caused by VFD are only
$0.002 \mathrm{MWh} / \mathrm{y}$ (accounting for only $0.02 \%$ of the overall EENS), which demonstrates the superiority of dual bus design and $\mathrm{N}+2$ redundancy mechanism. Compare single-bus system with dual-bus one, it can be seen that dual-bus system has better reliability than single-bus one. This is because dual busbar topology design can dramatically decrease the probability of the total plant loss.

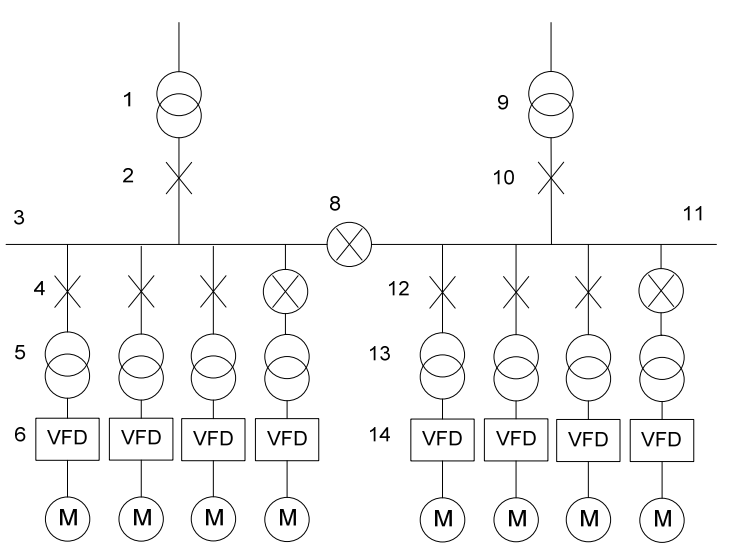

(a) Simplified SLD of water pumping station

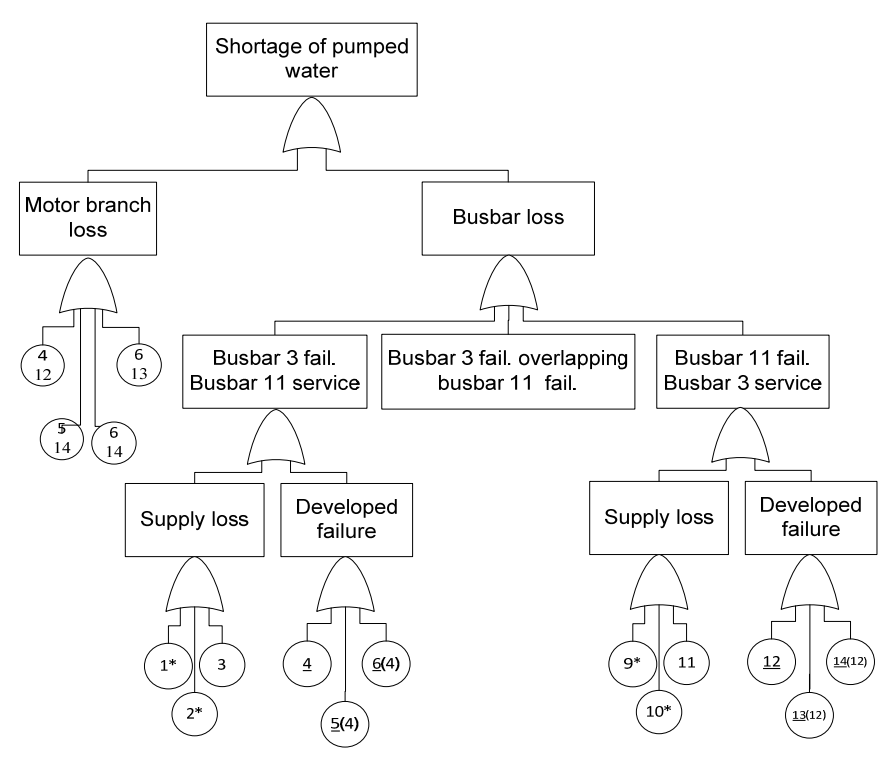

(b) Fault tree of water pumping station

Figure 1. Reliability case study for water pumping station.

Table 2. Reliability indices for dual bus water pumping station.

\begin{tabular}{|c|c|c|c|c|c|c|c|}
\hline & \multicolumn{2}{|c|}{ Busbar 3} & \multicolumn{2}{|c|}{ Busbar 11} & \multicolumn{3}{|c|}{ Busbar $3 \& 11$} \\
\hline & Failure event & $\mathrm{u}(\mathrm{h} / \mathrm{y})$ & Failure event & $\mathrm{u}(\mathrm{h} / \mathrm{y})$ & Failure event & $\mathrm{u}_{\text {backup fail. }}(\mathrm{h} / \mathrm{y})$ & $\mathrm{u}_{\text {back maint. }}(\mathrm{h} / \mathrm{y})$ \\
\hline \multirow{3}{*}{$\begin{array}{l}\text { Busbar loss } \\
\text { (power supply loss) }\end{array}$} & $1 *$ & 0.38 & $9^{*}$ & 0.38 & 1 & 28.02 & 8.02 \\
\hline & $2 *$ & 0.08 & $10^{*}$ & 0.08 & 2 & 5.25 & 0.25 \\
\hline & 3 & 0.05 & 11 & 0.05 & 3 & 0.05 & 0.05 \\
\hline \multirow{3}{*}{$\begin{array}{l}\text { Busbar loss } \\
\text { (motor path active failure loss) }\end{array}$} & $\underline{4}$ & 0.01 & $\underline{12}$ & 0.01 & $\underline{4}$ & 0.008 & 0.01 \\
\hline & $\underline{5}(4)$ & 0.00 & $\underline{13}(4)$ & 0.00 & $\underline{5}(4)$ & 0.00 & 0.00 \\
\hline & $\underline{6}(4)$ & 0.00 & $\underline{14}(4)$ & 0.00 & $\underline{6}(4)$ & 0.00 & 0.00 \\
\hline Bus outage duration (h/y) & \multicolumn{2}{|c|}{0.52} & \multicolumn{2}{|c|}{0.52} & \multicolumn{3}{|c|}{0.06} \\
\hline EENS $_{\text {busbar }}(\mathrm{MWh} / \mathrm{y})$ & \multicolumn{2}{|c|}{4.31} & \multicolumn{2}{|c|}{4.31} & \multicolumn{3}{|c|}{1.36} \\
\hline \multirow{3}{*}{ Motor loss } & 4 & 5.24 & $4^{*}$ & 0.07 & & & \\
\hline & 5 & 1.01 & $5^{*}$ & 0.00 & & & \\
\hline & 6 & 4.32 & $6^{*}$ & 0.00 & & & \\
\hline Motor outage duration (h/y) & \multicolumn{4}{|c|}{0.00} & & & \\
\hline EENS $_{\text {motor }}(\mathbf{M W h} / \mathbf{y})$ & \multicolumn{4}{|c|}{0.00} & & & \\
\hline EENS (MWh/y) & \multicolumn{4}{|c|}{9.97} & & & \\
\hline
\end{tabular}




\section{Conclusions}

In this paper, a generic reliability evaluation method for industry grid with VFD applications has been developed based on fault tree analysis method together with minimal cut set and set theory. Different topology design and redundancy mechanism have been considered in the algorithm design. Reliability of both single-bus and dualbus electrification schemes have been evaluated using the proposed method. Demonstrated by the two case studies, the proposed approach can not only quantify the reliability of a given system, but also differentiate the undesired outage caused by individual component, which is important to identify the weak point of the system for reliability improvement. Furthermore, it is also suitable for analyzing the reliability of industrial grids with multiple bus bars, or with other types of power electronic converter technology.

\section{REFERENCES}

[1] R. N. Allan, R. Billinton and M. F. Deoliveire, "Reliability Evaluation of the Auxiliary Electrical Systems of Power Stations," IEEE Transactions on Power Apparatus and Systems, Vol. 5, 1977, pp.1441-1449. doi:10.1109/T-PAS.1977.32472

[2] P. Zhou, H. G. Mou, Y. Liu and L. Zhang, "Reliability Evaluation for Electromechanical Fuze Based on Series Model and Parallel Model," Journal of Detection \& Control, Vo. 31, No. 6, 2009, pp. 51-54.

[3] R. Billinton, H. Chen and J. Zhou, "Generalized $n+2$ State System Markov Model for Station-Oriented Reliability Evaluation," IEEE Transactions on Power Systems, Vol. 12, No. 4, 1997, pp. 1511-1517. doi:10.1109/59.627850

[4] Y. Ou and L. Goel, "Using Monte Carlo Simulation for Overall Distribution System Reliability Worth Assessment," IEE Proceedings: Generation, Transmission and Distribution, Vol. 146, No. 5, 1999, pp. 535-540. doi:10.1049/ip-gtd: 19990542

[5] R. S. Chanda and P. K. Bhattacharjee, "A Reliability Approach to Transmission Expansion Planning Using Fuzzy Fault-Tree Model," Electric Power Systems Research, Vol. 45, No. 2, 1998, pp. 101-108. doi:10.1016/S0378-7796(97)01226-1

[6] http://spectrum.ieee.org/energy/environment/largest-solar -thermal-storage-plant-to-start-up 